\title{
Follow-up observations of Comet 17P/Holmes after its extreme outburst in brightness end of October 2007`
}

\author{
Markus Mugrauer $^{1}$, M. M. Hohle ${ }^{1,2}$, C. Ginski ${ }^{1}$, M. VAnKo $^{1}$, F. Freistetter $^{1}$ \\ 1 Astrophysikalisches Institut und Universitäts-Sternwarte Jena, Schillergässchen 2-3, 07745 Jena, Germany \\ 2 Max-Planck-Institut für extraterrestrische Physik, Giessenbachstrasse, 85748 Garching, Germany
}

Received; accepted; published online

\begin{abstract}
We present follow-up observations of comet 17/P Holmes after its extreme outburst in brightness, which occurred end of October 2007. We obtained 58 V-band images of the comet between October 2007 and February 2008, using the Cassegrain-Teleskop-Kamera (CTK) at the University Observatory Jena. We present precise astrometry of the comet, which yields its most recent Keplerian orbital elements. Furthermore, we show that the comet's coma expands quite linearly with a velocity of about $1650 \mathrm{~km} / \mathrm{s}$ between October and December 2007. The photometric monitoring of comet 17/P Holmes shows that its photometric activity level decreased by about 5.9 mag within 105 days after its outburst.
\end{abstract}

Key words: comets: individual (17/P Holmes)

(C)0000 WILEY-VCH Verlag GmbH \& Co. KGaA, Weinheim

\section{Introduction}

Comet $17 \mathrm{P} /$ Holmes is a short periodic comet, which was discovered by the British amateur astronomer Edwin Holmes close to the bright Andromeda galaxy M31 on November 6th 1892 (see Holmes 1892 or Krueger 1893). The comet passed through its perihelion already several month before its discovery, and had been in a much better condition for discovery before. Nevertheless, it was not detected which points out that the comet experienced most probably a strong outburst in brightness at the end of November 1892, which eventually enabled its visual discovery (Boss 1893). Barnard 1896, who carried out a follow-up study of the comet after its discovery, described that on November 9th 1892 17/P Holmes appeared to the naked eye brighter than the central part of M31, with a coma diameter of about 6 arcmin. During the next weeks the comet faded rapidly. However, mid of January 1893 a further outburst occurred and the magnitude of 17/P Holmes increased again to the 8th magnitude (Campbell 1893). Afterwards, the comet steadily became fainter, and no further successful observations were reported later than begin of April 1893.

Correspondence to: markus@astro.uni-jena.de

* Based on observations obtained with telescopes of the University Observatory Jena, which is operated by the Astrophysical Institute of the Friedrich-Schiller-University.
As member of the Jupiter family, close encounters between the gaseous giant planet Jupiter and 17/P Holmes take place occasionally. These encounters cause changes of the comet's orbit and have to be taken into account in the calculation of the comet's ephemerides. The first close encounter with Jupiter in the 20th century occurred 1908 (Kronk 2003). 17P/Holmes was observed successfully during its perihelion passages in 1892, 1899 and 1906. However, after its encounter with Jupiter, in 1908, the comet got lost and could not be detected during its expected following perihelion passages until that of 1964. Only by taking into account all astrometric measurements of the comet before it got lost after 1906, as well as the gravitational perturbations of Jupiter, it was possible to determine the altered orbital elements, and therefore to calculate more accurate ephemerides of the comet. Eventually, 17P/Holmes was recovered in 1964 and could be imaged during all its following perihelion passages.

The last time 17P/Holmes passed through its perihelion on May 4th 2007 (see section 3). The comet's brightness ranged between 14.1 and 16.5 mag between mid of July and begin of October 2007. On October 24th J. A. Henriquez Santana observed the comet and reported that it appeared much brighter ( $m \sim 8.4 \mathrm{mag}$ ) than just a few days before (Buzzi et al. 2007). The brightness of the comet continued to increase and finally reached about $2.5 \mathrm{mag}$, when $17 / \mathrm{P}$ Holmes appeared on the night sky as a yellow shiny point like object, easily visible to the naked eye. Hence, the brightness 
of the comet increased from about $16.5 \mathrm{mag}$ up to $2.5 \mathrm{mag}$, i.e. in total an outburst in brightness of $14 \mathrm{mag}$, the largest outburst of a comet observed so far.

Such a huge outburst in brightness could be caused by a meteorite impact on the comet's surface, which is however less probable, in particular if one takes into account that the comet showed already two outbursts before, end of 1892, and in early 1893. Another, more favorable scenario, is that, the comet was heated up by solar radiation during its perihelion passage, and underground layers were evaporated. Later on, the produced internal gas pressure disrupted the comet surface, which led to a dramatic emission of gas and dust, causing the outburst of the comet.

In this paper we present results of our follow-up observations of 17P/Holmes after its outburst. In section 2 we describe in detail all observations, which were carried out. In the following section "Data Analysis and Discussion" we present accurate astrometry of the comet, which allows the determination of its most recent orbital elements. Furthermore, we report on the evolution of the comet's coma, as well as on its photometric activity level after the outburst.

\section{Observations}

After its dramatic outburst in brightness end of October 2007, we started an imaging campaign of comet 17/P Holmes at the University Observatory Jena, using the CassegrainTelescope-Kamera (CTK) 1 . The comet could be observed with the CTK the first time on October 29th 2007, i.e. only five days after the discovery of the outburst. In total 58 images of the comet are taken, spread over 8 nights between October 2007 and February 2008. All observations are carried out in V-band and different integration times are used to avoid saturation of the CTK detector on the bright comet. In our first observing epoch integration times of only 20, and $30 \mathrm{~s}$ are applied, due to the high brightness of the comet (see Fig.11). Because of the fast decrease of the comet's brightness longer integration times of 60, and $300 \mathrm{~s}$ are chosen in the following observing epochs (see Fig. A1).

In order to remove the bias level, as well as the dark current from all our CTK images, at least three darkframes are always taken directly after the imaging of the comet. Cosmics are effectively suppressed in the median of these darkframes, and therefore the median of the darkframes is always subtracted from our CTK images. Afterwards, all images are flatfielded with skyflats, which are always taken in the same nights as our comet observations, either in evening or morning twilight. Furthermore, to remove bad pixels, we apply a badpixel correction to all dark+bias subtracted and flatfielded CTK images. We use a badpixel mask, which is created out of a series of domeflats, taken with different exposure times. The whole data-reduction is done with ESO-MIDAS and standard IRAF scripts.

All CTK images of comet 17/P Holmes are astrometrically calibrated using reference sources which are detected

\footnotetext{
1 See $\overline{\text { Mugrauer (2009) }}$ for a detailed description of this CCD imager.
}

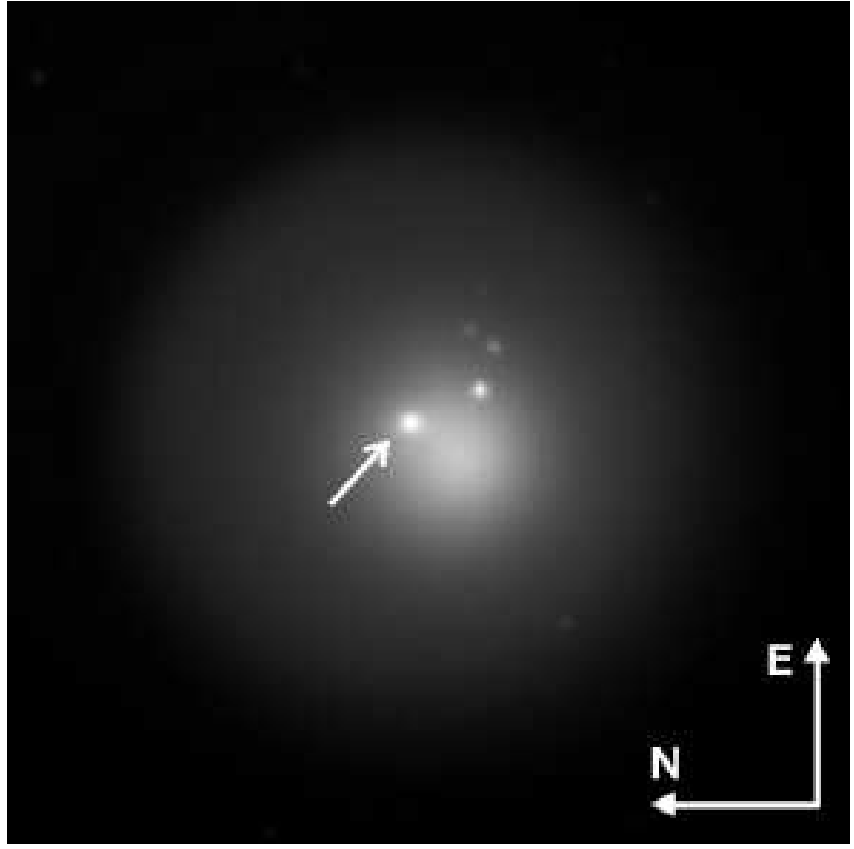

Fig. 1. Comet 17/P Holmes on October 29th 2007. The total integration time of this CTK image is $80 \mathrm{~s}$ in V-band. In order to avoid saturation of the CTK detector on the bright comet, four CTK images, each with 20 s of integration time, are taken, aligned, and finally added up. The pattern shown here is a display of the whole CTK image with $8.3^{\prime} \times 8.3^{\prime}$ field of view. The bright central part of the comet's coma is indicated with a white arrow. Several faint stars are visible, which shine through the outer thinner part of the coma, whose apparent angular radius measures already $\sim 3.6$ arcmin.

together with the comet in our CTK images, and are listed in the 2MASS Point Source Catalogue (Skrutskie et al. 2006). After calibration, the averaged uncertainty of the achieved absolute astrometry of our CTK images is only 0.28 arcsec relative to the 2MASS Point Source Catalogue. The astrometric calibration yields a pixelscale of the CTK detector of $2.2064 \pm 0.0003 \mathrm{arcsec} / \mathrm{pixel}$ for all observing epochs, i.e. the CTK field of view measures $37.7^{\prime} \times 37.7^{\prime}$.

\section{Data Analysis and Discussion}

In order to derive the most recent Keplerian elements of the orbit of comet 17/P Holmes, we measure the comet's position in all our 58 CTK images, using the gaussian centering algorithm "center/gauss" of ESO-MIDAS. The derived astrometry of the comet for all CTK observations is summarized in Tab. A1. We fit a Keplerian orbit to the measured comet's positions, and obtain its most recent orbital elements, which are summarized in Tab.1. The RMS error of the obtained orbital fit is only 0.663 arcsec. According to our astrometric measurements comet 17/P Holmes is on an eccentric ( $e=0.43277 \pm 0.00003$ ) orbit, and revolves around the Sun with a period of $P=6.8852 \pm 0.0004 \mathrm{yr}$. The comet's last perihelion passage took place on May 4th 2007 ( $\left.J D_{\text {Perihel }}=2454225.06 \pm 0.02\right)$ when it was separated from the Sun only by $q=2.0529 \pm 0.0001$ AU. The 
next perihelion passage of the comet is predicted for March 21 th $2014\left(J D_{\text {Perihel }}=2456738\right)$.

Table 1. Orbital elements of comet 17P/Holmes, derived by fitting an Keplerian orbit to our astrometry of the comet. The RMS error of the obtained orbital fit is 0.663 arcsec.

\begin{tabular}{ll}
\hline$J D_{\text {Perihel }}$ & $2454225.06 \pm 0.02$ \\
$a$ & $3.6192 \pm 0.0001 \mathrm{AU}$ \\
$e$ & $0.43277 \pm 0.00003$ \\
$i$ & $19.1119 \pm 0.0001^{\circ}$ \\
$\omega$ & $24.266 \pm 0.002^{\circ}$ \\
$\Omega$ & $326.8666 \pm 0.0009^{\circ}$ \\
\hline \hline
\end{tabular}

With the derived pixelscale of the CTK detector we can determine the extent of the coma of comet 17/P Holmes in all our CTK images. However, the whole extent of the comet's coma is imaged only in the first observing epoch in October 2007. Due to its fast expansion the size of the coma already exceeds the CTK field of view in all following observing epochs. In order to image completely also the widely expanded coma of comet 17/P Holmes, we obtained a mosaic of 9 CTK images on December $1^{\text {th }} 2007$. This mosaic, which covers a total field of view of $1.57^{\circ} \times 1.57^{\circ}$, is shown in Fig. A1 The total angular radius $\rho$ of the comet's coma, measured in both CTK observing runs in October and December 2007, is summarized in Tab.2. The same table also lists the comet's Earth distance $\Delta$, calculated with the derived orbital elements (see Tab.11).

Table 2. Expansion of the coma of comet 17P/Holmes. This table lists the total $(\rho)$ and the minimum angular radii $\left(\rho_{\min }\right)$ of the comet's coma for all observing epochs at which the radii can be measured in the CTK images. With the comet's Earth distance $\Delta$, derived from the determined orbital elements of the comet, the projected total radius $R$ of the coma, and its projected minimum radius $\mathrm{R}_{\min }$ can be derived.

\begin{tabular}{lccc}
\hline epoch & $\begin{array}{c}\rho \\
{[\operatorname{arcsec}]}\end{array}$ & $\begin{array}{c}\Delta \\
{[\mathrm{AU}]}\end{array}$ & $\begin{array}{c}R \\
{\left[10^{6} \mathrm{~km}\right]}\end{array}$ \\
\hline $29 / 10 / 07$ & $216 \pm 7$ & 1.6265 & $0.256 \pm 0.009$ \\
$01 / 12 / 07$ & $1293 \pm 12$ & 1.6967 & $1.595 \pm 0.015$ \\
\hline \hline epoch & $\rho_{\min }$ & $\Delta$ & $R_{\min }$ \\
& {$[\operatorname{arcsec}]$} & {$[\mathrm{AU}]$} & {$\left[10^{6} \mathrm{~km}\right]$} \\
\hline $29 / 10 / 07$ & $185 \pm 7$ & 1.6265 & $0.219 \pm 0.009$ \\
$27 / 11 / 07$ & $872 \pm 9$ & 1.6741 & $1.061 \pm 0.011$ \\
$28 / 11 / 07$ & $891 \pm 7$ & 1.6792 & $1.089 \pm 0.009$ \\
$01 / 12 / 07$ & $953 \pm 12$ & 1.6967 & $1.176 \pm 0.015$ \\
\hline \hline
\end{tabular}

Furthermore, Tab. 2 shows the projected radius $R$ of the coma, derived with its angular radius, and the comet's Earth distance for each observing epoch. With the given epoch difference between our two CTK observations in October and December 2007 ( $\Delta t=33.81$ days), we can finally determine the expansion velocity of the comet's coma and obtain $v_{\text {exp }}=1651 \pm 22 \mathrm{~km} / \mathrm{h}$.

Although, the whole coma is not imaged in all observing epochs, we can measure its minimum angular radius $\rho_{\min }$ in the first four epochs. The derived projected minimum radius of the comet's coma is listed in the second part of Tab.2 and is plotted for a range of time in Fig.2 The minimum radius of the coma expands quite linearly over time. A linear fit, which is illustrated with a dotted line in Fig.2 yields a slope of $1179.6 \pm 0.5 \mathrm{~km} / \mathrm{h}$. This increase of $R_{\min }$ can be considered as the minimum expansion velocity of the comet's coma. Due to the solar wind the coma of the comet should not expand with the same velocity in all directions. In particular, in direction towards the Sun we expect a minimum expansion velocity, resulting in a non-radial symmetric shape of the coma. If we assume that the coma expands linearly over time the outburst time can be derived directly from the linear fit of the expansion of the coma. According to the expansion of $R_{\text {min }}$ we obtain an outburst time $J D_{\text {out }}=2454394.8 \pm 2.3$, i.e. October $21.3 \pm 2.3$. If we use the expansion of the total radius of the coma we obtain an outburst time $J D_{\text {out }}=2454396 \pm 1$, i.e. October $22.5 \pm 1$. Hence, we can conclude that the outburst started only few days or even only few hours before it was discovered on October 24th.

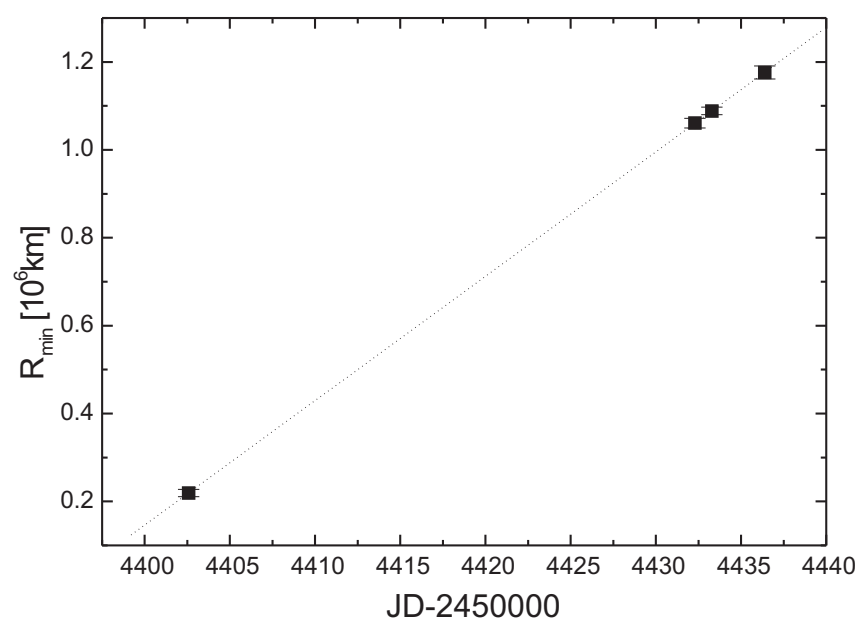

Fig. 2. The expansion of the coma of comet 17/P Holmes during the first month after its outburst in brightness. This plot shows the evolution of the minimum radius $R_{\min }$ of the comet's coma, between end of October until begin of December 2007. The minimum radius expands linearly over time with a velocity of $1179.6 \pm 0.5 \mathrm{~km} / \mathrm{s}$, the slope of this linear fit, which is illustrated as dotted line.

Beside the astrometric measurements described above, we also monitored the evolution of the comet's photometry after its outburst in brightness. In Tab. 3 we list the measured apparent V-band magnitudes of the bright central part of the comet's coma, which is also plotted for a range of time in Fig.3. For photometric calibration of the individual CTK images we always use several reference stars from the Hipparcos and Tycho catalogues (Perryman \& ESA 1997), which are imaged together with the comet and exhibit an accurate V-band photometry. With this method a photometric accuracy of $\Delta m=0.12 \mathrm{mag}$ can be achieved, in average. The apparent V-band brightness of the comet's central coma decreases by about 7.7 mag during 105 days between October 29th 2007 
and February 10th 2008. Because also the comet's Earth distance $\Delta$ increases significantly during this period (see Tab. 3), the change of distance has to be taken into account to obtain the true photometric activity level of the comet. Therefore, we determine the absolute magnitude $M_{\mathrm{V}}$ of the comet's central coma, i.e. its apparent magnitude at a fixed distance of $1 \mathrm{AU}$. The absolute magnitude can be derived with the measured apparent magnitude of the coma and the comet's Earth distance $\Delta$. Tab. 3] shows the derived absolute magnitude of the comet's central coma for all observing epochs. The accuracy of the derived absolute photometry is dominated by the accuracy of the measured apparent magnitudes.

Table 3. Photometry of the central coma of comet 17/P Holmes for all CTK observing epochs. The apparent V-band magnitude of the coma is listed together with its absolute magnitude, derived with its apparent photometry and the calculated comet's Earth distance $\Delta$.

\begin{tabular}{lcrcr}
\hline epoch & JD-2450000 & $\begin{array}{c}V \\
{[\mathrm{mag}]}\end{array}$ & $\begin{array}{c}\Delta \\
{[\mathrm{AU}]}\end{array}$ & $\begin{array}{c}\mathrm{M}_{\mathrm{V}} \\
{[\mathrm{mag}]}\end{array}$ \\
\hline $29 / 10 / 07$ & 4402.58 & $8.06 \pm 0.10$ & 1.6265 & $7.00 \pm 0.10$ \\
$27 / 11 / 07$ & 4432.30 & $12.83 \pm 0.06$ & 1.6741 & $11.71 \pm 0.06$ \\
$28 / 11 / 07$ & 4433.29 & $12.77 \pm 0.13$ & 1.6792 & $11.65 \pm 0.13$ \\
$01 / 12 / 07$ & 4436.39 & $13.01 \pm 0.11$ & 1.6967 & $11.86 \pm 0.11$ \\
$07 / 02 / 08$ & 4504.31 & $15.43 \pm 0.16$ & 2.5433 & $13.40 \pm 0.16$ \\
$08 / 02 / 08$ & 4505.29 & $15.30 \pm 0.11$ & 2.5597 & $13.26 \pm 0.11$ \\
$09 / 02 / 08$ & 4506.29 & $15.44 \pm 0.15$ & 2.5764 & $13.39 \pm 0.15$ \\
$10 / 02 / 08$ & 4507.27 & $15.77 \pm 0.13$ & 2.5930 & $13.71 \pm 0.13$ \\
\hline \hline
\end{tabular}

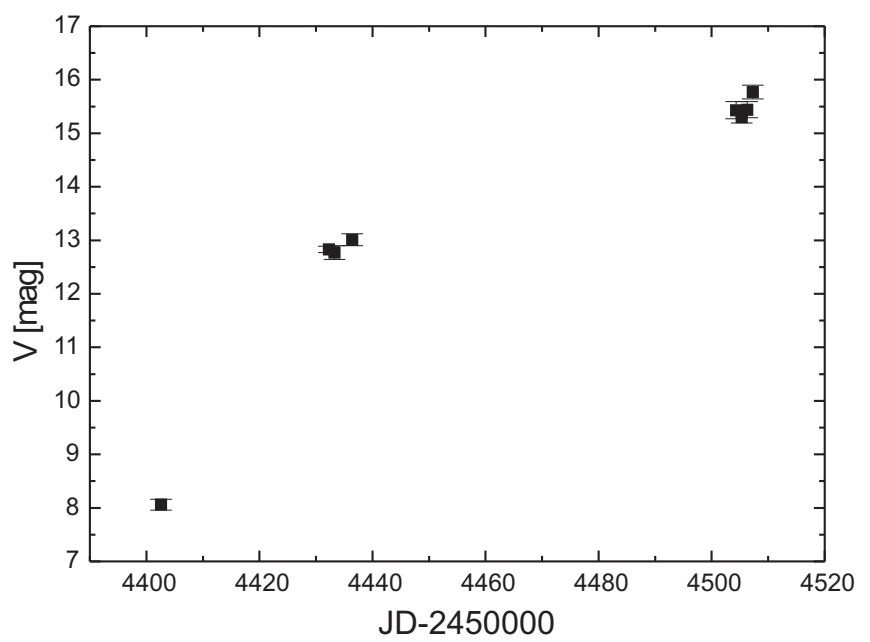

Fig. 3. This plot shows the apparent V-band magnitude $V$ of the central coma of comet 17P/Holmes for all CTK observing epochs between October 2007 and February 2008. Between 105 days its apparent brightness decreases by $\Delta V=7.71 \pm 0.16 \mathrm{mag}$.

During our follow-up observations the absolute magnitude of the central coma of comet 17/P Holems decreased by $\Delta M_{V}=6.71 \pm 0.16 \mathrm{mag}$, see Tab. 3. However, because of the increase of the comet's Sun distance in the same span of time, the comet's natural activity level is also expected to decrease, due to lower solar radiation at larger solar distance.

Whipple (1978) studied the natural brightness variation of comets before and after their perihelion passage which follows in general a $r^{-n}$ relation $(n>2 \sqrt{2}$. For short periodical comets, essential for those of the Jupiter family, he reported comparable $n$-values before and after their perihelion passages, namely $5.0 \pm 1.4$, and $4.61 \pm 0.91$, respectively. Taking into account the decrease of the dust production rate $Q_{D}$ at wider Sun distances these empirical relations agree well with the theoretical approach derived by Fernández et al. (1999), which yielded a $Q_{D}^{2} \cdot r^{-4}$ dependency.

As comet 17/P Holmes already passed through its perihelion in May 2007, i.e. before our CTK observations, we use the $r^{4.61 \pm 0.91}$ relation of Whipple (1978) to approximate the expected natural decrease of the absolute brigntness of the comet's central coma. The difference between the decrease of the absolute magnitude of the coma and its expected natural decrease, finally yields the true decrease of the comet's outburst activity level. Within the 105 days of our followup observations of comet 17/P Holmes, its distance to the Sun increased from 2.46 to $2.89 \mathrm{AU}$. Therefore, according to the relation reported by Whipple (1978), we expect a natural decrease of the absolute magnitude of the comet's central coma of only $\Delta M_{V \star}=0.82 \pm 0.16 \mathrm{mag}$, while the observed decrease is $\Delta M_{V}=6.71 \pm 0.16 \mathrm{mag}$. Hence, the outburst activity level of 17/P Holmes significantly decreases by $\Delta M=5.89 \pm 0.23 \mathrm{mag}$, i.e. a factor between 183 to 281 within the given span of time. Tab. 4 shows the observed decrease of the absolute magnitude of the comet's central coma, its expected natural magnitude decrease, as well as the derived decrease of the comet's outburst activity level, for all CTK observing epochs.

Table 4. Photometric variability of the central coma of comet 17/P Holmes since its outburst in brightness. The comet's Sun distance $r$ is calculate with the derived orbital elements of the comet. $\Delta M_{V}$ is the observed decrease of the absolute magnitude of the comet's central coma since the first CTK observing epoch. $\Delta M_{V \star}$ is the expected natural decrease of its absolute magnitude due to the change of the comet's Sun distance. Their difference, $\Delta M$, yields the decrease of the comet's outburst activity level.

\begin{tabular}{ccccc}
\hline epoch & $\begin{array}{c}r \\
{[\mathrm{AU}]}\end{array}$ & $\begin{array}{c}\Delta M_{V} \\
{[\mathrm{mag}]}\end{array}$ & $\begin{array}{c}\Delta M_{V \star} \\
{[\mathrm{mag}]}\end{array}$ & $\begin{array}{c}\Delta M \\
{[\mathrm{mag}]}\end{array}$ \\
\hline $29 / 10 / 07$ & 2.4553 & 0 & 0 & 0 \\
$27 / 11 / 07$ & 2.5738 & $4.71 \pm 0.12$ & $0.24 \pm 0.05$ & $4.47 \pm 0.13$ \\
$28 / 11 / 07$ & 2.5778 & $4.65 \pm 0.16$ & $0.24 \pm 0.05$ & $4.41 \pm 0.17$ \\
$01 / 12 / 07$ & 2.5906 & $4.86 \pm 0.15$ & $0.27 \pm 0.05$ & $4.59 \pm 0.16$ \\
$07 / 02 / 08$ & 2.8799 & $6.40 \pm 0.19$ & $0.80 \pm 0.16$ & $5.60 \pm 0.25$ \\
$08 / 02 / 08$ & 2.8841 & $6.26 \pm 0.15$ & $0.81 \pm 0.16$ & $5.45 \pm 0.22$ \\
$09 / 02 / 08$ & 2.8885 & $6.39 \pm 0.18$ & $0.81 \pm 0.16$ & $5.58 \pm 0.24$ \\
$10 / 02 / 08$ & 2.8927 & $6.71 \pm 0.16$ & $0.82 \pm 0.16$ & $5.89 \pm 0.23$ \\
\hline \hline
\end{tabular}

\footnotetext{
${ }^{2}$ The expected natural decrease of brightness of a non-active body should follow a $r^{-2}$ relation, which is also expected for comets but only at very large solar distances $r$.
} 
Acknowledgements. M. Vanko acknowledges support from the EU in the FP6 MC ToK project MTKD-CT-2006-042514. M. M. Hohle acknowledges partial support from DFG in the SFB/TR-7 Gravitation Wave Astronomy. We make use of data products from the Two Micron All Sky Survey, which is a joint project of the University of Massachusetts and the Infrared Processing and Analysis Center/California Institute of Technology, funded by the National Aeronautics and Space Administration and the National Science Foundation, as well as the SIMBAD and VIZIER databases, operated at CDS, Strasbourg, France.

\section{References}

Barnard, E. E. 1896, ApJ, 3,41

Buzzi, L., Muler, G., Kidger, M., Henriquez Santana, J. A., Naves, R., Campas, M., Kugel, F., \& Rinner, C. 2007, IAUC, 8886, 1

Boss, L. 1893, PASP, 5, 94

Campbell, W. W. 1893, PASP, 5, 93

Fernández, J. A., Tancredi, G., Rickman, H., \& Licandro, J. 1999, A\&A, 352, 327

Holmes, E. 1892, The Observatory, 15, 441

Kronk, G. W. 2003, Cometography, by Gary W. Kronk, pp. 852. ISBN 0521585058. Cambridge, UK: Cambridge University Press, December 2003.,

Krueger, A. 1893, AN, 131, 133

Skrutskie, M. F., et al. 2006, AJ, 131, 1163

Perryman, M. A. C., \& ESA 1997, ESA Special Publication, 1200, Mugrauer M., 2009, AN, arXiv:0903.4116

Whipple, F. L. 1978, Moon and Planets, 18, 343

\section{Appendix A: Appendix}

Table A1. CTK astrometry of comet 17/P Holmes. The averaged uncertainty of the absolute CTK astrometry is 0.28 arcsec relative to the $2 \mathrm{MASS}$ point source catalogue.

\begin{tabular}{lllcc}
\hline date & & & RA & $\begin{array}{c}\text { Dec } \\
\text { yyyy }\end{array}$ \\
mm & dd.ddddd & hh:mm:ss.ss & dd:mm:ss.s \\
\hline 2007 & 10 & 29.05340 & $03: 49: 11.41$ & $+50: 23: 56.9$ \\
2007 & 10 & 29.06932 & $03: 49: 10.41$ & $+50: 23: 59.9$ \\
2007 & 10 & 29.07134 & $03: 49: 10.31$ & $+50: 24: 00.3$ \\
2007 & 10 & 29.07337 & $03: 49: 10.16$ & $+50: 24: 00.6$ \\
2007 & 10 & 29.07610 & $03: 49: 10.00$ & $+50: 24: 01.2$ \\
2007 & 10 & 29.07817 & $03: 49: 09.87$ & $+50: 24: 01.7$ \\
2007 & 10 & 29.08020 & $03: 49: 09.75$ & $+50: 24: 01.9$ \\
2007 & 10 & 29.08216 & $03: 49: 09.64$ & $+50: 24: 02.6$ \\
2007 & 10 & 29.08413 & $03: 49: 09.52$ & $+50: 24: 02.6$ \\
2007 & 10 & 29.08610 & $03: 49: 09.39$ & $+50: 24: 03.5$ \\
2007 & 10 & 29.10067 & $03: 49: 08.48$ & $+50: 24: 06.0$ \\
2007 & 11 & 27.79446 & $03: 14: 41.66$ & $+49: 20: 45.1$ \\
2007 & 11 & 27.79563 & $03: 14: 41.62$ & $+49: 20: 44.4$ \\
2007 & 11 & 27.79681 & $03: 14: 41.56$ & $+49: 20: 44.7$ \\
2007 & 11 & 27.79792 & $03: 14: 41.44$ & $+49: 20: 44.6$ \\
2007 & 11 & 27.79941 & $03: 14: 41.37$ & $+49: 20: 44.6$ \\
2007 & 11 & 27.80903 & $03: 14: 40.75$ & $+49: 20: 40.8$ \\
2007 & 11 & 28.74995 & $03: 13: 42.78$ & $+49: 13: 56.8$ \\
\hline
\end{tabular}

\begin{tabular}{llccc}
2007 & 11 & 28.75102 & $03: 13: 42.72$ & $+49: 13: 55.6$ \\
2007 & 11 & 28.75209 & $03: 13: 42.63$ & $+49: 13: 54.1$ \\
2007 & 11 & 28.75329 & $03: 13: 42.58$ & $+49: 13: 53.5$ \\
2007 & 11 & 28.75436 & $03: 13: 42.52$ & $+49: 13: 53.2$ \\
2007 & 11 & 28.75543 & $03: 13: 42.45$ & $+49: 13: 53.2$ \\
\hline date & & & RA & Dec \\
yyyy & mm & dd.ddddd & hh:mm:ss.ss & dd:mm:ss.s \\
\hline 2007 & 11 & 28.75661 & $03: 13: 42.44$ & $+49: 13: 52.9$ \\
2007 & 11 & 28.75769 & $03: 13: 42.34$ & $+49: 13: 52.7$ \\
2007 & 11 & 28.75875 & $03: 13: 42.18$ & $+49: 13: 51.3$ \\
2007 & 11 & 28.75983 & $03: 13: 42.18$ & $+49: 13: 51.3$ \\
2007 & 11 & 28.78810 & $03: 13: 40.38$ & $+49: 13: 39.9$ \\
2007 & 11 & 28.78918 & $03: 13: 40.26$ & $+49: 13: 39.4$ \\
2007 & 11 & 28.79024 & $03: 13: 40.21$ & $+49: 13: 39.5$ \\
2007 & 11 & 28.79131 & $03: 13: 40.14$ & $+49: 13: 38.7$ \\
2007 & 11 & 28.79238 & $03: 13: 40.06$ & $+49: 13: 38.4$ \\
2007 & 11 & 28.79949 & $03: 13: 39.62$ & $+49: 13: 35.8$ \\
2007 & 11 & 28.80056 & $03: 13: 39.61$ & $+49: 13: 35.0$ \\
2007 & 11 & 28.80163 & $03: 13: 39.50$ & $+49: 13: 34.6$ \\
2007 & 11 & 28.80271 & $03: 13: 39.45$ & $+49: 13: 33.8$ \\
2007 & 11 & 28.80500 & $03: 13: 39.26$ & $+49: 13: 32.6$ \\
2007 & 11 & 28.80608 & $03: 13: 39.24$ & $+49: 13: 32.3$ \\
2007 & 11 & 28.80714 & $03: 13: 39.19$ & $+49: 13: 32.0$ \\
2007 & 11 & 28.80822 & $03: 13: 39.03$ & $+49: 13: 30.9$ \\
2007 & 11 & 28.80928 & $03: 13: 39.01$ & $+49: 13: 30.7$ \\
2007 & 11 & 28.81071 & $03: 13: 38.94$ & $+49: 13: 29.8$ \\
2007 & 11 & 28.81177 & $03: 13: 38.88$ & $+49: 13: 29.4$ \\
2007 & 11 & 28.81285 & $03: 13: 38.81$ & $+49: 13: 29.2$ \\
2007 & 11 & 28.81391 & $03: 13: 38.73$ & $+49: 13: 28.3$ \\
2007 & 11 & 28.81498 & $03: 13: 38.67$ & $+49: 13: 28.0$ \\
2007 & 12 & 01.88733 & $03: 10: 39.08$ & $+48: 50: 00.5$ \\
2007 & 12 & 01.88840 & $03: 10: 39.03$ & $+48: 50: 00.1$ \\
2007 & 12 & 01.88948 & $03: 10: 38.93$ & $+48: 49: 59.2$ \\
2007 & 12 & 01.89079 & $03: 10: 38.87$ & $+48: 49: 58.9$ \\
2007 & 12 & 01.89186 & $03: 10: 38.83$ & $+48: 49: 57.9$ \\
2008 & 02 & 07.80910 & $03: 23: 24.35$ & $+39: 28: 15.7$ \\
2008 & 02 & 07.81730 & $03: 23: 23.72$ & $+39: 27: 58.8$ \\
2008 & 02 & 08.79417 & $03: 24: 27.82$ & $+39: 23: 20.2$ \\
2008 & 02 & 09.78072 & $03: 25: 32.20$ & $+39: 18: 30.7$ \\
2008 & 02 & 09.78676 & $03: 25: 32.52$ & $+39: 18: 29.2$ \\
2008 & 02 & 09.79315 & $03: 25: 33.07$ & $+39: 18: 27.2$ \\
2008 & 02 & 10.77179 & $03: 26: 37.98$ & $+39: 13: 46.1$ \\
\hline & & & &
\end{tabular}




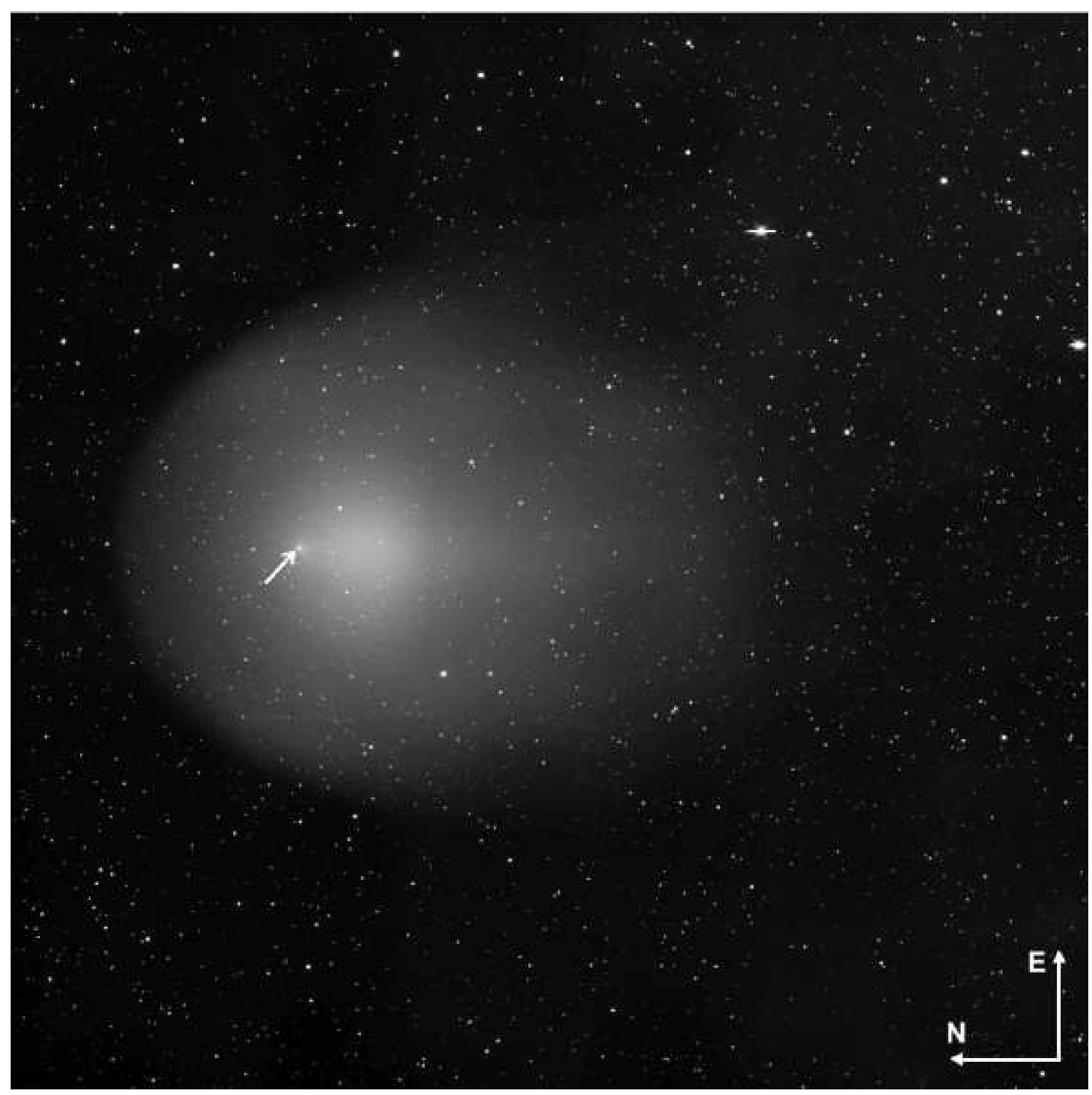

Fig. A1. Comet 17P/Holmes on Dec $1^{\text {th }} 2007$. This pattern shows a V-band mosaic, composed of 9 CTK images each with an integration time of $300 \mathrm{~s}$. The field of view covered by this mosaic is $1.57^{\circ} \times 1.57^{\circ}$, showing the huge extent of the comet's coma about one month after the outburst in brightness. The position of the comet's central coma is indicated by a white arrow in the CTK mosaic. 